\title{
Enxerto venoso intercoronariano na revascularização de artéria coronária comprometida por dissecção de aorta tipo I
}

Carlos Alberto C. ABREU FILHO ${ }^{*}$, Richard H. CABRAL*, Alexandra P. GASPAR*, Luís Alberto DALLAN $^{*}$, Sérgio Almeida de OLIVEIRA*, Adib D. JATENE*

\begin{abstract}
ABREU FILHO, C. A. C.; CABRAL, R. H.; GASPAR, A. P.; DALLAN, L. A.; OLIVEIRA, S. A.; JATENE, A. D. - Enxerto venoso intercoronariano na revascularização de artéria coronária comprometida por dissecção de aorta tipo I. Rev. Bras. Cir. Cardiovasc., 10 (1): 56-60, 1995.

RESUMO: Paciente de 39 anos, portador de hipertensão arterial não controlada, submetido a cirurgia para tratamento de disseç̧ăo aguda de aorta tipo I. Devido ao grande envolvimento do óstio coronário direito pela dissecção aórtica, optou-se pela ressecção da porção comprometida do vaso, com interposição de tubo năo valvulado de Dacron na aorta ascendente, suspensāo da valva aórtica e ligadura do óstio da artéria coronária direita. Como não havia presença de aterosclerose no ramo interventricular anterior e na artéria coronária direita, decidimos realizar um enxerto venoso entre as artérias. O enxerto intercoronário apresentou bom funcionamento e todo o território dependente da coronária direita manteve boa contratilidade. O paciente recebeu alta hospitalar em boas condiçōes clínicas, com função miocárdica preservada.
\end{abstract}

DESCRITORES: Aorta, dissecção, cirurgia. Miocardio, revascularização, cirurgia.

\section{INTRODUÇÃO}

A dissecção de aorta constitui complicação grave em pacientes portadores de hipertensão arterial. Sua manifestação pode ou não estar associada à degeneração estrutural da parede arterial. As principais manifestações clínicas incluem dor torácica aguda de forte intensidade, associada ou não a sintomas neurológicos, gastrintestinais, respiratórios, hipotensão ou choque. As complicações mais freqüentes são insuficiência valvar aórtica, comprometimento do fluxo coronariano, acidente vascular cerebral e rotura da parede da aorta. O tratamento preconizado é cirúrgico, procurando conter a hemorragia conseqüente à rotura do vaso, suspenção ou troca valvar aórtica (caso comprometida) e suspensão da porção lesada da aorta por enxerto tubular, sempre que possível acima dos óstios das artérias coronárias, com a finalidade de preservar - fluxo sangũíneo coronariano. Caso esses óstios estejam envolvidos pela dissecção, torna-se necessário o implante coronariano no tubo interposto, ou a realização de um enxerto entre o tubo e a coronária envolvida. No presente caso, ao invés da ponte entre o tubo de Dacron e a coronária comprometida, realizamos um enxerto venoso intercoronariano, entre a artéria coronária esquerda (ramo interventricular anterior), cujo óstio não estava envolvido pela dissecção, e a coronária direita.

\section{RELATO DO CASO}

Paciente do sexo masculino, 39 anos, com antecedente de hipertensão arterial não tratada há

\footnotetext{
Trabalho realizado no Instituto do Coração do Hospital das Clínicas da Faculdade de Medicina da Universidade de São Paulo, São Paulo, SP, Brasil.

* Do Instituto do Coração do Hospital das Clínicas da Faculdade de Medicina da Universidade de São Paulo.

Recebido para publicação em março de 1995.

Endereço para correspondência: Luis Alberto Dallan. Av. Dr. Enéas de Carvalho Aguiar, 44. Divisão Cirúrgica. CEP 05403-000 São Paulo, SP, Brasil.
} 
ABREU FILHO, C. A. C.; CABRAL, R. H.; GASPAR, A. P.; DALLAN, L. A.; OLIVEIRA, S. A.; JATENE, A. D. - Enxerto venoso intercoronariano na revascularização de artéria coronária comprometida por dissecção de aorta tipo I. Rev. Bras. Cir. Cardiovasc., 10 (1): 56-60, 1995.

dois anos e episódios de dor em hemitórax direito associados a amaurose. Deu entrada no Pronto Socorro do Instituto do Coração do Hospital das Clínicas da Faculdade de Medicina da Universidade de São Paulo apresentando dor torácica em região precordial, de início súbito, de forte intensidade, com duração de aproximadamente 1 hora e irradiação para região interescapular. O paciente negava fatores desencadeantes da dor. Ao exame físico, apresentava-se em bom estado geral, hipertenso (PA 180 $\times 160$ ), com assimetria de pulsos entre os membros superiores e inferiores, e presença de sopro diastólico $(++/ 6+)$ em foco aórtico na ausculta cardíaca.

A cineangiocoronariografia evidenciou presença de dissecção de aorta tipo I associada a insuficiência aórtica leve, com artérias coronárias sem obstrução mas com hipocinesia difusa do ventrículo esquerdo. Confirmado o diagnóstico, indicou-se tratamento cirúrgico.

O paciente foi submetido a cirurgia através de esternotomia mediana, com instalação da circulação extracorpórea, hipotermia corpórea moderada $\left(28^{\circ} \mathrm{C}\right)$ e proteção miocárdica com solução cardioplé-

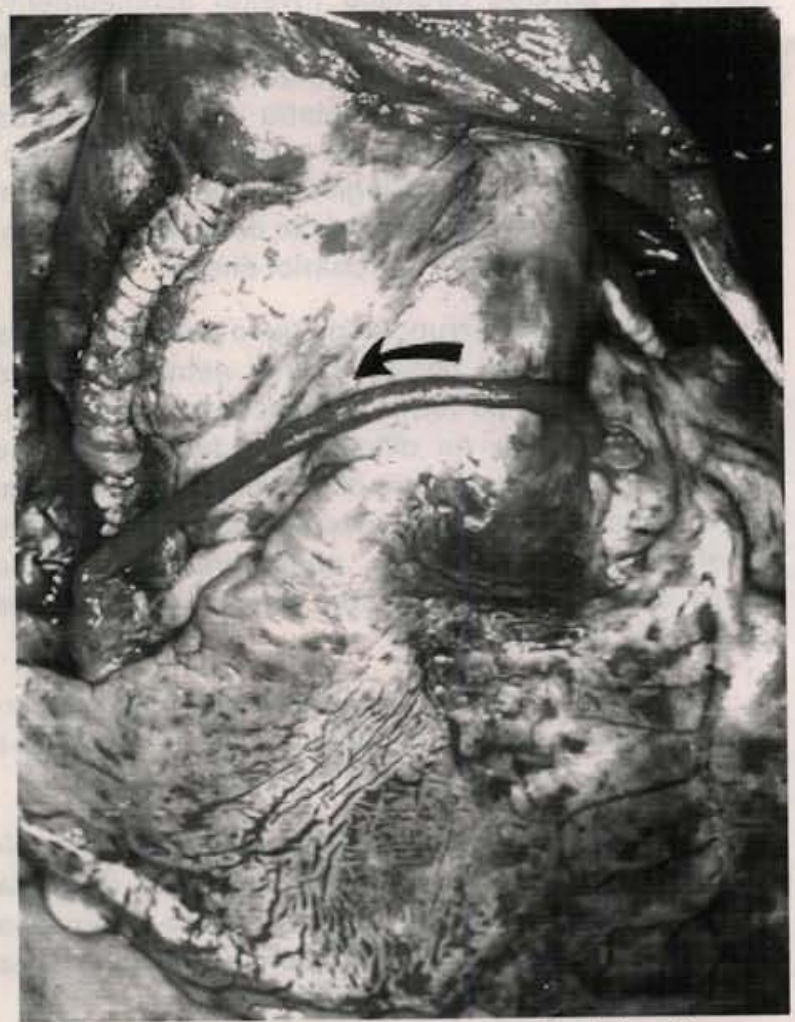

Fig. 1 - Aspecto final da correção da dissecção aórtica. 0 excesso de tecido da aorta ascendente foi chuleado sobre o tubo de Dacron, visando a melhor hemostasia. Destaca-se a ponte de veia safena entre a artéria interventricular anterior $e$ a artéria coronária direita (seta). gica injetada nos óstios coronários. Após pinçamento da aorta, foi realizada aortotomia longitudinal. $O$ achado intra-operatório foi de dissecção aórtica tipo I, comprometendo a aorta ascendente e envolvendo o óstio coronário direito. Optou-se pela ressecção da porção comprometida do vaso, com interposição de tubo de Dacron não valvulado e pré-coagulado, de $34 \mathrm{~mm}$ de diâmetro, ligadura do óstio da artéria coronária direita e suspensão da valva aórtica. As camadas da parede da aorta foram aproximadas e reforçadas com cola biológica (GRF) e sutura com pontos simples de Prolene 5-0. A interposição do tubo de Dácron foi realizada através de sutura contínua com fio Mononylon 4-0 e 5-0 e reforço com cola gel. Devido a dificuldades técnicas, tanto para se implantar a coronária direita no tubo interposto como também para realizar uma ponte entre essas duas estruturas, decidiu-se revascularizar essa coronária através de ponte de safena posicionada entre a artéria interventricular anterior e a coronária direita (Figuras 1 e 2). Após a saída de CEC e estabilização hemodinâmica, o paciente foi enviado à Unidade de Recuperação Pós-Operatória. Evoluiu bem, tendo tido alta hospitalar assintomático. A ecocardiografia de controle demonstrou boa contratilidade cardiaca e discreta insuficiência valvar aórtica.

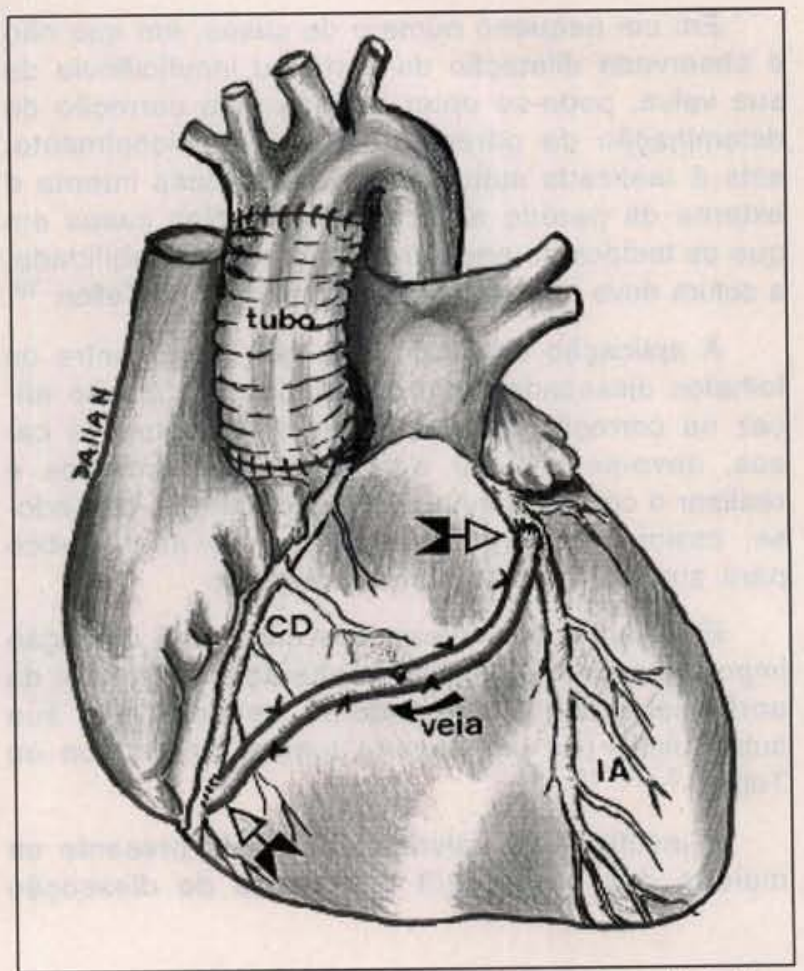

Fig. 2 - Desenho esquemático da tática cirúrgica. O sentido do fluxo sanguíneo pela ponte de safena, interventricular anterior (1A) coronária direita $(C D)$ está indicado pela seta. 
ABREU FILHO, C. A. C.; CABRAL, R. H.; GASPAR, A. P.; DALLAN, L. A.; OLIVEIRA, S. A.; JATENE, A. D. - Enxerto venoso intercoronariano na revascularização de artéria coronária comprometida por dissecção de aorta tipo I. Rev. Bras, Cir. Cardiovasc., 10 (1): 56-60, 1995.

\section{COMENTÁRIOS}

A dissecção constitui uma afecção grave da aorta, merecendo especial atenção devido à elevada taxa de mortalidade imediata $1,9,10,12,13$.

As complicações eventualmente observadas são decorrentes da progressão da dissecção: isquemia miocárdica, tamponamento cardíaco por rotura intrapericárdica, acidente vascular cerebral e insuficiência valvar aórtica.

O tratamento da dissecção de aorta ascendente é basicamente cirúrgico. Caso não se institua tratamento após o episódio inicial, a mortalidade gira em torno de $100 \%{ }^{19}$. A terapêutica clínica isolada (baseada na administração de hipotensores e betabloqueadores) tem se mostrado insatisfatória, poia a mortalidade é muito elevada, chegando aos $80 \% 1,9,10,12,13$. Por outro lado, qualquer que seja a medida terapêutica adotada (clínica ou cirúrgica), resulta em mortalidade precoce de $30 \%$.

A terapêutica cirúrgica faz-se necessária, na maioria dos casos, tendo como objetivo a substituição da aorta ascendente, corrigindo sua dissecção e aliviando a insuficiência aórtica.

A tática cirúrgica vai depender do grau de comprometimento da aorta e da coexistência ou não de suas principais complicaçōes: o envolvimento dos óstios coronários e a insuficiência valvar aórtica ${ }^{14}, 19$.

Em um pequeno número de casos, em que não é observada dilatação da aorta ou insuficiência de sua valva, pode-se optar apenas pela correção da delaminação da parede aórtica. Tradicionalmente, esta é realizada suturando-se as lâminas interna e externa da parede aórtica entre si. Nos casos em que os tecidos se encontram com maior friabilidade, a sutura deve ser reforçada com retalhos de Teflon ${ }^{19}$.

A aplicação direta de cola gel (GRF) entre os folhetos dissecados tem constituído um auxílio eficaz na correção de sua delaminação. Nesses casos, deve-se realizar a remoção dos trombos $e$ realizar o correto alinhamento dos folhetos obtendose, assim, inclusive a suspensão do anel aórtico para sua posição anatômica original.

Entretanto, na maioria dos casos, há dilatação importante, com significativa alteração estrutural da aorta ascendente, tornando-se necessária a sua substituição por um enxerto tubular de Dacron ou Teflon 3, 14, 19, 20.

A insuficiência valvar aórtica está presente na maioria dos casos (2/3 dos casos de dissecção aórtica tipo I) $1-3,5,7,11-17,19,20$ e pode ser tratada cirurgicamente, seja de forma conservadora $3,14,20$, ou através de substituição da valva por prótese mecânica ou biológica ${ }^{19}$.

A maioria dos autores advoga a conduta conservadora e afirma que a simples suspensão da valva com a remodelação de seu anel é suficiente para a correção da insuficiência $3,14,20$. Além disso, os autores salientam as desvantagens relacionadas à troca valvar, tais como sua possível disfunção ou os problemas inerentes à anticoagulação prolongada.

Quando uma das situações estiver presente, ou quando a dilatação aórtica se iniciar junto ao anel valvar, uma boa alternativa cirúrgica é a técnica proposta por Bentall ${ }^{14},{ }^{19}$. Ela consiste na inserção de um tubo de Dacron contendo uma válvula interposta entre o anel aórtico e a aorta ascendente, e a circulação coronariana pode ser reconstituída através da implantação dos óstios coronários no enxerto, ou através de ponte de safena entre este e as artérias coronárias.

No caso relatado, a ausência de história pregressa de cardiopatia ou doença do tecido conjuntivo permitiu optar pelo tratamento conservador da valva aórtica. $\mathrm{O}$ achado cirúrgico revelou acometimento com dilatação importante da porção ascendente da aorta e comprometimento do óstio coronário direito. A estratégia cirúrgica consistiu na correção da delaminação dos folhetos da parede aórtica, através do uso de cola biológica e seu reforço com sutura contínua, e na interposição do tubo de Dacron, com ligadura do óstio coronário direito.

Em vista da interrupção do fluxo sangüíneo para a artéria coronária direita, houve necessidade de revascularização do território miocárdico dependente do vaso. Dada as dificuldades técnicas de se implantar o óstio coronário direito no tubo de Dacron e a ausência de aterosclerose nas coronárias direita e interventricular anterior, consideramos a ponte entre elas uma boa opção, diferentemente dos pacientes coronariopatas.

A técnica utilizada para revascularização miocárdica foi eficaz, tendo o paciente apresentado boa evolução no pós-operatório imediato e tardio.

Consideramos que a interrupção do fluxo sangüineo coronariano decorrente da dissecção da aorta exige correção cirúrgica. É apresentada alternativa técnica para casos em que apenas um dos óstios coronários esteja comprometido. 
ABREU FILHO, C. A. C.; CABRAL, R. H.; GASPAR, A. P.; DALLAN, L. A.; OLIVEIRA, S. A.; JATENE, A. D. - Enxerto venoso intercoronariano na revascularização de artéria coronária comprometida por dissecção de aorta tipo I. Rev. Bras. Cir. Cardiovasc., 10 (1): 56-60, 1995.

\section{RBCCV 44205-259}

ABREU FILHO, C. A. C.; CABRAL, R. H.; GASPAR, A. P.; DALLAN, L. A.; OLIVEIRA, S. A.; JATENE, A. D. - Intercoronary venous graft in revascularization of the coronary artery involved by aortic dissection type I. Rev. Bras. Cir. Cardiovasc., 10 (1): 56-60, 1995.

ABSTRACT: A thirty nine-year-old patient presenting arterial hypertension was operated upon for type I acute aortic dissection. Due to important alterations in the ascendent aorta with involvement of the right coronary ostium, the surgical procedure included resection of the segment of aorta containing the intimal tear and replacement with a Dacron prosthesis, suspension of the aortic valve and ligadure of the right coronary ostium. Technical difficulties brought the necessity of a different approach for the myocardium revascularization. Instead of the traditional described procedures, an intercoronary venous graft was put between the anterior interventricular artery (branch of the left coronary artery) and the right coronary artery. The intercoronary graft presented a good flow since the begining, keeping a good myocardial contratility. Patient left the hospital in a good clinical condition, with preserved myocardial function. Intercoronary graft should be an efficient alternative for revascularization of a coronary artery involved by aortic dissections.

DESCRIPTORS: Aortic dissection, surgery. Myocardial revascularization, surgery

\section{REFERÊNCIAS BIBLIOGRÁFICAS}

1 APPLEBAUM, A.; KARP, R. B.; KIRKLIN, J. W. Ascending vs descending aortic dissections. Ann. Surg., 183: 296-300, 1976.

2 BACHET, J.; GIGOU, F.; LAURIAN, C. - Four-year clinical experience with the gelatin-resorcine-formol biological glue in acute aortic dissection. J. Thorac. Cardiovasc. Surg., 83: 212-217, 1982.

3 CACHERA, J. P.; VOUHÉ, P. R.; LOISANCE, D. Y.; MENU, P.; POULAIN, H.; BLOCH, G.; VASILE, N.; AUBRY, P.; GALEY, J. J. - Surgical management of acute dissections involving the ascending aorta: early and late results in 38 patients. J. Thorac. Cardiovasc. Surg., 823: 576-584, 1981.

4 CAMARANO, G. P.; MOREIRA, L. F. P.; STOLF, N. A. G.; BARRETO, A. C. P.; BELLOTTI, G.; PILEGGI, F.; JATENE, A. D. - Avaliação da insuficiência aórtica na dissecção de aorta. Importância da ecocardiografia intra-operatória na conservação da valva: relato de caso. Arq. Bras. Cardiol., 52: 345-348, 1989.

5 DeBAKEY, M. E.; HENLY, W. S.; COOLEY, D. A.; CRAWFORD, E. S.; BEALL, A. C. - Surgical management of dissecting aneurysm of the aorta. J. Thorac. Cardiovasc. Surg., 49: 130, 1965.

ENIA, F. - Dissecazione e insufficienza aortica. G. Ital. Cardiol., 21: 1041-1042, 1991 (Carta ao autor).
7 FABIANI, J. N.; JEBARA, V. A.; DELOCHE, A.; STEPHAN, Y.; CARPENTIER, A. - Use of surgical glue without replacement in the treatment of type $A$ aortic dissection. Circulation, 80 (Supl. 1): 264-268, 1989.

8 GRUNKEMEIER, G.; LAMBERT, L.; BONCHEK, L.; STARR, A. - An improved statistical method for assessing the results of operation. Arm. Thorac. Surg., 20: 289-298, 1975.

9 HARRIS, P. D.; MALM, J. R.; BIGGER Jr., J. T. - Followup studies of acute dissecting aortic aneurysms managed with antihypertensive agents. Circulation, 35 (Supl. 2): 183-187, 1967.

10 HIRST Jr., A. E.; JOHNS Jr., V. S.; KIME Jr., S. W. Dissecting aneurysm of the aorta: a review of 505 cases. Medicine, 37: 217-279, 1958.

11 LAENNEC, R. T. H. - De l'ascultation médiate, ou traité du diagnostic des maladies des poumons et du coeur, Paris, Chez J-A Brossand, et J-S Chaudé, vol. 1, p. 411, 1819.

12 LINDSAY Jr., J.; HURST, J. W. - Clinical features and prognosis in dissecting aneurysm of the aorta. A reappraisal. Circulation, 35: 880-888, 1967.

13 McFARLAND, J.; WIRLESTON, J. T.; DINSMORE, R. E.; AUSTEN, W. G.; BUCKLEY, M. J.; SANDERS, C. A.; DeSANCTIS, E. W. - The medical treatment 
ABREU FILHO, C. A. C.; CABRAL, R. H.; GASPAR, A. P.; DALLAN, L. A.; OLIVEIRA, S. A.; JATENE, A. D. - Enxerto venoso intercoronariano na revascularização de artéria coronária comprometida por dissecção de aorta tipo I. Rev. Bras. Cir. Cardiovasc., 10 (1): 56-60, 1995.

of dissecting aortic aneurysms. New Engl. J. Med., 286: $115-119,1972$.

14 MAZZUCOTELLI, J.P.; DELEUZE, P. H.; BAUFRETON, C.; DUVAL, A. M.; HILLION, M. L.; LOISANCED, Y.; CACHERA, J. P. - Preservation of the aortic valve in acute aortic dissection: long-term echocardiographic assessment and clinical outcome. Ann. Thorac. Surg., 55: 1513-1517, 1993.

15 MILLER, D. C. - Surgical management of aortic dissections: indications, perioperative management and long-term results. In: DOROGHAZI, R. M. \& SLATER, E. E. (eds.) Aortic dissection. New York, McGraw-Hill, 193-244, 1983.

16 MORGAGNI, G. B. - DeSedibus et Causis Morborum per Anatomen Indagatis Libri Quinque. Venetiis, ex Typog Remondiuiana, 1761.
17 SAVIOTTI, M. - Insufficienza valvolare aortica in corso di dissezione aortica: descrizione di un caso insolito. Casi clinici. G. Ital. Cardiol., 21: 845-848, 1991.

18 SLATER, E. E. \& DeSANCTIS, R. W. - The clinical recognition of dissecting aortic aneurysm. Am. J. Med., 60: 625-633, 1975.

19 STOLF, N. A. G. \& ZERBINI, E. J. - Dissecção da aorta torácica. In: RAIA, A. A. \& ZERBINI, E. J. (eds.) Clínica Cirúrgica Alípio Correa Netto, 4 ed. São Paulo, Sarvier, 1994. p. 772-778.

20 WEINSCHELBAUM, E. E.; SCHAMUN, C.; CARAMUTTI, V.; TACCHI, H.; CORS, J.; FAVALORO, R. G. Surgical treatments of acute type A dissecting aneurysm, with preservation of the native aortic valve and use of biologic glue: follow-up to six years. J. Thorac. Cardiovasc. Surg., 103: 369-374, 1992. 